\title{
Introducing a Phenomenon of Single Junction Multiple Band-gap Solar Cell Compared to Single Junction or Multi Junction Solar Cell to Achieve High Efficiency
}

\author{
Nahid Akter ${ }^{1}$, M. Moshiur Rahman ${ }^{2}$, Shirin Akter ${ }^{3}$
}

${ }^{1}$ Dept. of Computer Science \& Telecommunication Engineering, Noakhali Science \& Technology University, BANGLADESH

${ }^{2}$ Department of Physics, Jahangirnagar University, BANGLADESH

${ }^{3}$ Accelerator Facilities Division, Bangladesh Atomic Energy Commission, BANGLADESH

\begin{abstract}
Besides cost, the most fundamental issue in assessing photovoltaic solar cells is efficiency. The benefits of single-junction solar cells are easier to manufacture, simpler and less expensive to make. However, they are less efficient than multi-junction solar cells. But when different semiconductors are stacked in layers to create multiple junctions, the atoms in the lower layers must not be blocked by the atoms in the upper layers or the sunlight passes by them instead of striking them. That means higher efficiency multijunction solar cells are difficult to make. From the periodic table, group III-V and group II-VI produce the alloys of direct band-gap semiconductor. If group $\mathrm{V}$ anions are partially replaced with nitrogen atom or group VI element is partially replaced with oxygen atom then new semiconductor material is produced that respond to a wider spectrum of frequencies within sunlight. It will make single-junction multiple band-gap solar cell which is more efficient and less expensive to manufacture.
\end{abstract}

Keywords: Band-gap, lattice, HMAs, BAC, electro-negativity.

\section{INTRODUCTION}

Band-gap fundamentally limits the colors that a solar cell can convert to electricity. Charge cannot flow in either a completely full or a completely empty band, but doping a semiconductor provides extra electrons or positively charged "holes" that can carry a current. Photons with just the right energy, the color of light that matches the band-gap create electron-hole pairs and allow current to flow across the junction between positively and negatively doped layers. In direct band-gap semiconductor, light of the right energy does not vibrate the lattice thus creates electron-hole pairs more efficiently than the indirect band-gap semiconductors. All direct-band-gap semiconductors combine elements of group III with group V or group II with group VI of the periodic table (Shan etal, 1999). 


\section{Single Junction Solar Cell}

Photons with energy lower than the band gap escape unabsorbed, photons with higher energy are absorbed, but most of their energy is wasted as heat. Crystalline silicon, the leading solar cell material, has a band gap of only about $1.1 \mathrm{eV}$, most solar photons are much more energetic. Crystalline-silicon solar cells are about 25 percent efficient at best (Meillaud etal, 2006).

\section{Multi-Junction Solar Cell}

Different materials with different band gaps can be stacked to capture photons with a wider range of energies. In a multi-junction solar cell, the top junction captures highenergy photons, while others pass through to the lower-band-gap junctions below. If aluminium gallium arsenide, which has a band gap of about $1.7 \mathrm{eV}$, could be layered with crystalline silicon, theoretically the resultant cell would be 50 percent efficient. Unfortunately these materials can't be stacked; matching materials with different crystal lattices is difficult and often impossible. The most efficient multi-junction cells yet made of two-junction cells with about 30 percent efficiency (Meillaud etal, 2006).

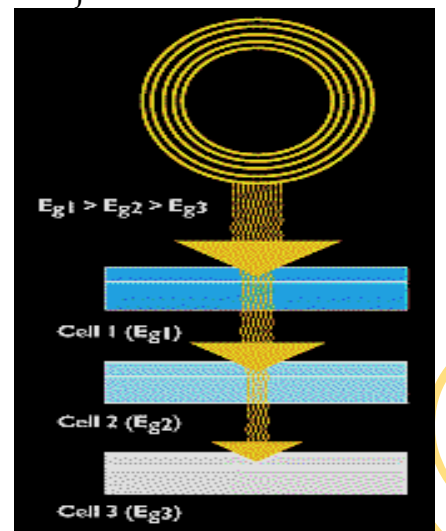

Fig 1: Materials with different band-gaps can be stacked in multi-junction cells where each layer responds to a different energy of sunlight

\section{Single Junction Multiple Band-gap Phenomenon}

Introducing impurities with the right electronic properties into a semiconductor one can make a single junction solar cell that absorbs more photons with different energies. That means a single junction of the material may be able to respond to virtually the entire solar spectrum. This isn't a multi-junction material rather a multi-gap material a single semiconductor with multiple band gaps (Preuss, 2004).

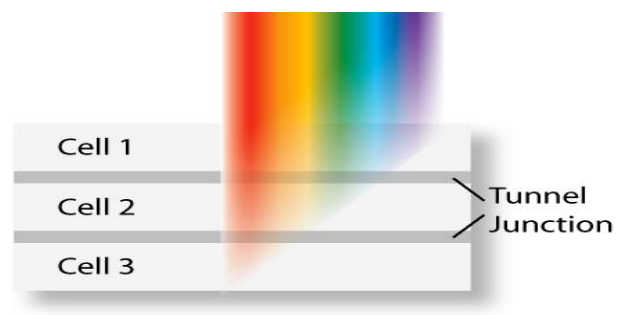

(a)

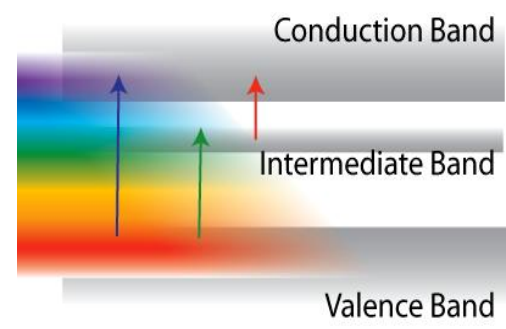

(b) 
Fig 2: (a) Multi-junction solar cell where each layer is a different alloy with a different band gap, which responds to a different frequency of sunlight. (b) Multi-gap solar cell has only a single layer of material, but multiple band gaps allow it to respond to a range of different frequencies (Walukiewicz, 2007)

\section{Design Technique of Multiband Structure}

The design of semiconductor alloys with an intermediate band within the energy gap provides multiband structure. The design of the material is based on band anti-crossing (BAC) model of highly mismatched semiconductor alloys (HMAs). Group III-N-V alloys in which group V anions are partially replaced with $\mathrm{N}$ or group II-O-VI alloys in which group VI element is replaced with $\mathrm{O}$ are the well known examples of the HMAs. Or HMAs is formed when anions are partially replaced with isovalent elements with distinctly different electro-negativities and/or atomic radii, e.g. III- $\mathrm{N}_{x}-\mathrm{V}_{1-\mathrm{x}}, \mathrm{II}-\mathrm{O}_{\mathrm{x}}-\mathrm{VI}{ }_{1-\mathrm{x}}$. The values of electro-negativities $(\mathrm{X})$ with respect to atomic radii for group V-VI are shown in the table 1(Preuss, 2004) below.

Table 1: Electro negativities (X) and atomic radii (R) (Walukiewicz, 2007)

\begin{tabular}{|c|c|c|}
\hline IV & V & VI \\
\hline \multirow{3}{*}{$\begin{array}{c}C \\
X=2.6\end{array}$} & $\mathbf{N}$ & $\mathrm{O}$ \\
\hline & $X=3.0$ & $X=3.4$ \\
\hline & $\mathrm{R}=0.075 \mathrm{~nm}$ & $\mathrm{R}=0.073 \mathrm{~nm}$ \\
\hline \multirow{3}{*}{$\begin{array}{c}\mathrm{Si} \\
\mathrm{X}=1.9\end{array}$} & $\mathbf{P}$ & $S$ \\
\hline & $X=2.2$ & $X=2.6$ \\
\hline & $\mathrm{R}=0.12 \mathrm{~nm}$ & $\mathrm{R}=0.11 \mathrm{~nm}$ \\
\hline \multirow{3}{*}{$\begin{array}{c}\mathrm{Ge} \\
X=2.0\end{array}$} & As & Se \\
\hline & $X=2.2$ & $X=2.6$ \\
\hline & $\mathrm{R}=0.13 \mathrm{~nm}$ & $\mathrm{R}=0.12 \mathrm{~nm}$ \\
\hline \multirow{3}{*}{$\begin{array}{c}\text { Sn } \\
X=2.0\end{array}$} & $\mathrm{Sb}$ & Te \\
\hline & $X=2.1$ & 2.1 \\
\hline & $\mathrm{R}=0.14 \mathrm{~nm}$ & $\mathrm{R}=0.14 \mathrm{~nm}$ \\
\hline
\end{tabular}

Alloy presents the opportunity to tune the optical and electronic properties of a semiconductor simply by altering its composition and with advancements in thin film growth techniques. It is now possible to fabricate single-phase materials composed of highly immiscible compounds. Among these new alloys, semiconductors known as highly mismatched alloys (HMAs) undergo a dramatic change in their optical and electrical properties upon the addition of only a few percent of the alloying species.

Highly mismatched alloys include those compound semiconductors wherein the anion species has been partially replaced by an isovalent impurity of much different electronegativity or size. Examples include $\operatorname{GaN}_{x} A s_{1-x}, \mathrm{GaN}_{x} P_{1-x}, \mathrm{ZnO}_{x} \mathrm{Te}_{1-x}$, $G a S b_{x} A s_{1-x}, G a B i_{x} A s_{1-x}$, and $Z n T e_{x} S_{1-x}$. The electronic structure of HMAs is determined by band anti-crossing model (BAC).

\section{BAC MODEL}

The band-gap of the HMAs alloys has an unusually strong dependence on the composition as well as pressure and in some instances possesses an extra impurityderived band. In order to explain these unusual properties band anti-crossing (BAC) model takes into account of the interaction of the impurity species with the host crystal. Although, these impurities are electrically neutral but introduce localized states that 
undergo an anti-crossing interaction with the delocalized states of the host crystal. When the impurity species has a much greater electronegativity than that of the host anion, the defect states of the impurity atoms are often located near the conduction band edge of the host. Electrons are localized near the impurity sites, and n-like states that interact. As a result, the conduction band is split into $\mathrm{E}+$ and $\mathrm{E}^{-}$sub-bands. Conversely, when the impurity species has a much smaller first ionization energy than the host anion, the defect states of these metallic atoms often lie near the valence band edge of the host. Holes are localized at the impurities sites, and the p-like states interact. Consequently, the anticrossing interaction splits the valence band into heavy hole, light hole and spin-orbit splitoff -derived $\mathrm{E}+$ and $\mathrm{E}^{-}$bands.

BAC model described by the following equation

$$
E_{ \pm}=\frac{1}{2}\left[\left(E^{C}(k)+E^{L}\right) \pm \sqrt{\left[\left(E^{C}(k)-E^{L}\right)\right]^{2}+4 C^{2}{ }_{N M} \cdot x}\right]
$$

Where, $E^{\mathrm{C}}(\mathrm{k})$ and $E^{\mathrm{L}}$ are the energies of the extended state and of the $\mathrm{N}$ level respectively. $C_{\mathrm{NM}}$ (Kin etal, 2003) is the matrix element of the term describing the interaction between localized $\mathrm{N}$ states and the extended states.

$E_{+}$and $E_{-}$are the energy eigenvalues of the BAC Hamiltonian used to describe the level repulsion.

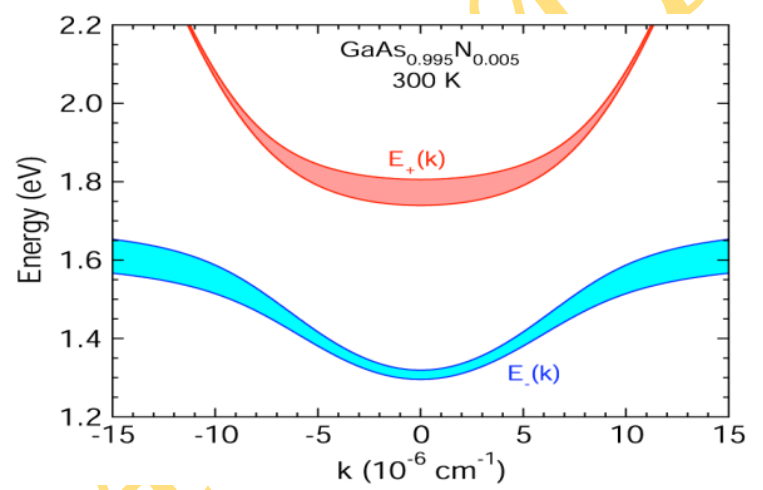

Fig 3: Local level splits conduction band (Walukiewicz, 2007)

\section{Dilute NitRides AND Dilute Oxides}

Group II-VI dilute oxide (II-O-VI) semiconductors with the anions partially replaced by highly electronegative isoelectronic $\mathrm{O}$ atoms are a direct analog of the III-V diluted nitrides. Group II$\mathrm{O}-\mathrm{VI}$ alloys in which highly electronegative $\mathrm{O}$ partially replaces the group VI element show behaviors that are similar to those of III-N-V alloys. For example, a dramatic O-induced reduction of the band gap has been reported in $C d_{1-y} M n_{y} O_{x} T e_{1-x}$ and $Z n O_{x} S e_{1-x}$. The electronic band structure of the dilute oxides is determined by an anti-crossing interaction between localized states of $\mathrm{O}$ and the extended states of the semiconductor matrix.

In most instances, e.g. $\mathrm{N}$ in $\mathrm{Ga}-\mathrm{As}$ or $\mathrm{O}$ in $\mathrm{Cd}-\mathrm{Te}$, the localized states are located within the conduction band and consequently a relatively wide lower sub-band is formed. This is manifested as a reduction of the energy band gap. A narrow band can be formed only if the localized states occur well below the conduction band edge. The BAC model predicts that the anti-crossing interaction of the $\mathrm{O}$ states with the extended conduction band states in the $Z n_{1-y} M n_{y} T e$ will lead to the formation of a narrow band of intermediate states. 
With multiple band gaps that fall within the solar energy spectrum, $Z n_{1-y} M n_{y} O_{x} T e_{1-x}$ is extremely well suited for the high efficiency multi-band single-junction solar cells.

Dilute III-V nitrides and II-VI oxides are HMAs in which small amount of the V or VI hosts are substituted by highly electronegative $\mathrm{N}$ and $\mathrm{O}$ respectively. Conversely, when a larger, more metallic element substitutes the anions, such as in $G a N_{1-x} A s_{x}, Z n S_{1-x} T e_{x}$ and $Z n S e_{1-x} T e_{x}$ , the localized impurity states will be located near the host valence band edge and will induce a similar anti-crossing interaction that will cause the valence band to restructure. This type of behavior is also expected in dilute $G a S b_{x} A s_{1-x}$ and $G a B i_{x} A s_{1-x}$ alloys. The electronic structure of these HMAs can be well described the valence band anti-crossing (VBAC) model. The band-gap reduction is primarily the result of an upward shift of the Ga-As related heavy and light hole bands induced by an anti-crossing interaction with those of the impurity atoms. This interaction also modifies the position of the spin-orbit split-off band, and as a result the spin-orbit splitting energy rises with $x($ Kin etal, 2003).

\section{Efficiency Comparison}

Photons with energy lower than the band gap escape unabsorbed; photons with higher energy are absorbed, but most of their energy is wasted as heat. A single-junction cell made from silicon first created by Bell Laboratories and converted about six percent of the light that struck it into electricity. Crystalline silicon, the leading solar cell material has a band gap of only about $1.1 \mathrm{eV}$. So it is about 25 percent efficient at best.

In a multi-junction solar cell, the top junction captures high-energy photons, while others pass through to the lower-band-gap junctions below. If aluminum gallium arsenide which has a band gap of about $1.7 \mathrm{eV}$ could be layered with crystalline silicon the resultant cell would be 50 percent efficient. Unfortunately these materials can't be stacked. Matching materials with different crystal lattices is difficult and often impossible. The most efficient multi-junction cells yet made are of two-junction cells with about 30 percent efficiency (Preuss, 2004).

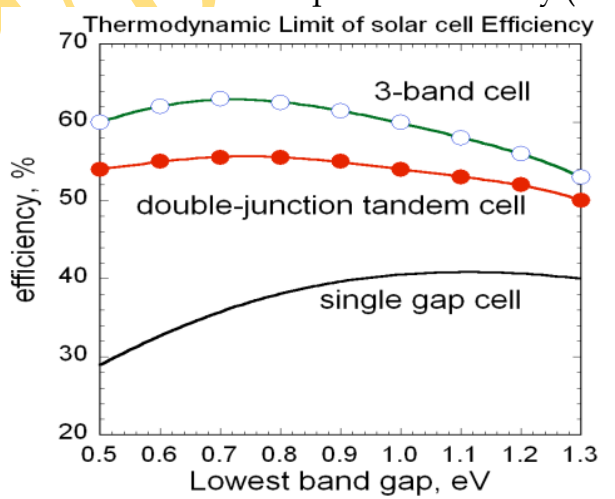

Fig 4: Efficiency improvement for single junction multiband solar cell rather than single cell or tandem cell (Walukiewicz, 2007)

In some materials, however, the band-anti-crossing model predicts that the impurity atoms will produce a narrow band well below the conduction band. The Berkeley Lab researchers first proposed indium gallium nitride for a full-spectrum solar cell because the crystal lattice of all the different layers is the same. Indium gallium nitride (In-Ga-N) would respond to different wavelengths of light if the proportions of indium and gallium in the alloy were adjusted. Research continues on In-Ga-N for satellite applications, 
although it has proved difficult to make a practical p-type version of the material. Again adding oxygen impurities to the II-VI alloy zinc manganese tellurium (Zn-Mn-Te) would produce well defined and widely split band gaps so that it could achieve more than 50 percent efficiency with the simplest possible physical structure (Preuss, 2004).

\section{CONCLUSION}

The introduction of states in a semiconductor band gap presents an alternative to multijunction designs for improving the power conversion efficiency of solar cells. The intermediate band allowing absorption of photons at three different energy levels, corresponding to the three different band-gap. A single junction cell with a properly located band of intermediate states could achieve power conversion efficiencies higher than those for optimized double-junction tandem cells. For II-VI oxide multi-band semiconductor synthesized using the combination of oxygen ion implantation and pulsed laser melting. In case of $\mathrm{ZnTe}$, a narrow intermediate band formed by the incorporation of $\mathrm{O}$. In addition to the $\mathrm{O}$ content, $\mathrm{Mn}$ alloying can also be used to adjust the energy level positions. With multiple band gaps that fall within the solar energy spectrum, $Z n_{1-x} M n_{x} O_{y} T e_{1-y}$ is well suited for the high efficiency intermediate band solar cells. This highly non-equilibrium technique allowed for the synthesis of ZnMnOTe alloys with up to 3\% of Te replaced with $\mathrm{O}$ atoms. Ion implantation and pulsed-laser melting technique has also used for the formation of supersaturated substitutional alloys of Si and group III-V elements.

\section{REFERENCES}

F. Meillaud, A. Shah, C. Droz, E. Vallat-Sauvain, C. Miazza, "Efficiency limits for single junction \& tendem solar cells", Solar Energy Materials and Solar Cells 90, issues 18-19, 2952-2959, 2006.

Kin Man Yu, Wladek Walukiewicz, Wei Shan, and Jeff Beeman; "Diluted II-VI oxide semiconductors with multiple band gaps, Physical Review Letters, 12 December 2003.

Paul Preuss, Berkley Lab Science Beat: A Step Closer to the Optimum Solar Cell;; March 2004.

W. Shan,W.Walukiewicz, J.W. Ager III, E. E. Haller, J. F. Geisz, D. J. Friedman, J.M. Olson, and S. R. Kurtz, Phys. Rev. Lett. 82, 1221(1999).

W. Walukiewicz, “New material for high efficiency solar cells”, ALS Workshop, 10/5/2007,.

Websites:

http://emat-solar.lbl.gov/research/band-anti-crossing-model

http:/ / emat-solar.lbl.gov/research/dilute-nitrides

http://emat-solar.lbl.gov/research/dilute-oxides

http:/ /emat-solar.lbl.gov/research/pulsed-laser-melting-plm

\section{ABC-JAR!!!}

"Speedy publication service, Online archives, Paperless, web-based peer review system, Open access policy, Indexing in world known citation databases, Global circulation, Broad international readership and authorship, Online submission system, Minimum publication charge" 